\begin{tabular}{|c|l|}
\hline Title & Geometry-driven shift in the Tomonaga Luttinger exponent of deformed cylinders \\
\hline Author(s) & Shima, Hiroyuki; Y oshioka, Hideo; Onoe, Jun \\
\hline Citation & $\begin{array}{l}\text { Physical Review B, 79(20), 201401 } \\
\text { https://doi.org/L0.1103/PhysRevB.79.201401 }\end{array}$ \\
\hline Issue Date & 2009-05 \\
\hline Doc URL & http://hdl.handle.net/2115/38684 \\
\hline Rights & ○ 2009 The American Physical Society \\
\hline Type & article \\
\hline File Information & 79-20_201401.pdf \\
\hline
\end{tabular}

Instructions for use 


\title{
Geometry-driven shift in the Tomonaga-Luttinger exponent of deformed cylinders
}

\author{
Hiroyuki Shima, ${ }^{1, *}$ Hideo Yoshioka, ${ }^{2}$ and Jun Onoe ${ }^{3}$ \\ ${ }^{1}$ Department of Applied Physics, Graduate School of Engineering, Hokkaido University, Sapporo 060-8628, Japan \\ ${ }^{2}$ Department of Physics, Nara Women's University, Nara 630-8506, Japan \\ ${ }^{3}$ Department of Nuclear Engineering and Research Laboratory for Nuclear Reactors, Tokyo Institute of Technology, 2-12-1 Ookayama, \\ Meguro, Tokyo 152-8550, Japan
}

(Received 10 March 2009; published 15 May 2009)

\begin{abstract}
We demonstrate the effects of geometric perturbation on the Tomonaga-Luttinger liquid (TLL) states in a long, thin, and hollow cylinder whose radius varies periodically. The variation in the surface curvature inherent to the system gives rise to a significant increase in the power-law exponent of the single- particle density of states. The increase in the TLL exponent is caused by a curvature-induced potential that attracts low-energy electrons to region that has large curvature.
\end{abstract}

DOI: 10.1103/PhysRevB.79.201401

PACS number(s): 73.21.Hb, 02.40.-k, 03.65.Ge, 71.10.Pm

Studying the quantum mechanics of a particle confined to curved surfaces has been a problem for more than 50 years. The difficulty arises from operator-ordering ambiguities, ${ }^{1}$ which permit multiple consistent quantizations for a curved system. The conventional method used to resolve the ambiguities is the confining-potential approach., ${ }^{2,3}$ In this approach, the motion of a particle on a curved surface (or, more generally, a curved space) is regarded as being confined by a strong force acting normal to the surface. Because of the confinement, quantum excitation energies in the normal direction are raised far beyond those in the tangential direction. Hence, we can safely ignore the particle motion normal to the surface, which leads to an effective Hamiltonian for propagation along the curved surface with no ambiguity.

It is well known that the effective Hamiltonian involves an effective scalar potential whose magnitude depends on the local surface curvature. ${ }^{2-5}$ As a result, quantum particles confined to a thin curved layer behave differently from those on a flat plane, even in the absence of any external field (except for the confining force). Such curvature effects have gained renewed attention in the last decade, mainly, because of the technological progress that has enabled the fabrication of low-dimensional nanostructures with complex geometry. ${ }^{6,8-11,7,12-14}$ From the theoretical perspective, many intriguing phenomena pertinent to electronic states, ${ }^{15-22}$ electron diffusion, ${ }^{23}$ and electron transport ${ }^{24-27}$ have been suggested. In particular, the correlation between surface curvature and spin-orbit interaction ${ }^{28,29}$ as well as with the external magnetic field ${ }^{30-32}$ has been recently considered as a fascinating subject.

Most of the previous works focused on noninteracting electron systems, though few have focused on interacting electrons $^{33}$ and their collective excitations. However, in a low-dimensional system, Coulombic interactions may drastically change the quantum nature of the system. Particularly noteworthy are one-dimensional systems, where the Fermiliquid theory breaks down so that the system is in a Tomonaga-Luttinger liquid (TLL) state. $^{34}$ In a TLL state, many physical quantities exhibit a power-law dependence stemming from the absence of single-particle excitations near the Fermi energy; this situation naturally raises the question as to how geometric perturbation affects the TLL behaviors of quasi-one-dimensional curved systems. Peanut- shaped $\mathrm{C}_{60}$ polymers ${ }^{35,36}$ and $\mathrm{MoS}_{2}$ hollow nanotubes ${ }^{37}$ are exemplary materials to be considered for studying TLL behaviors; they are thin, long, and hollow tubules whose radius is periodically modulated along the tube axis. Hence, the periodic surface curvature intrinsic to the systems will produce sizable effects on their TLL properties if they exhibit one-dimensional metallic properties.

In this Rapid Communication, we examined the curvature effects on the TLL states in quantum hollow cylinders with a periodically varying radius. We demonstrate that the presence of a curvature-induced potential can yield a significant increase in the power-law exponent $\alpha$ of the single-particle density of states $n(\omega)$ near the Fermi energy $E_{F}$; i.e., $n(\omega)$ $\propto\left|\hbar \omega-E_{F}\right|^{\alpha} .38,39$ The geometric conditions required for the shift in $\alpha$ to be observable are within the realm of laboratory experiments, which implies that our predictions can be verified with existing materials.

We first considered noninteracting spinless electrons confined to a general two-dimensional curved surface $S$ embedded in a three-dimensional Euclidean space. A point $\boldsymbol{p}$ on $S$ is represented by $\boldsymbol{p}=\left[x\left(u^{1}, u^{2}\right), y\left(u^{1}, u^{2}\right), z\left(u^{1}, u^{2}\right)\right]$, where $\left(u^{1}, u^{2}\right)$ is a curvilinear coordinate spanning the surface and $(x, y, z)$ are the Cartesian coordinates in the embedding space. Using the notation $\boldsymbol{p}_{i} \equiv \partial \boldsymbol{p} / \partial u^{i}(i=1,2)$, we introduced the following quantities $g_{i j}=\boldsymbol{p}_{i} \cdot \boldsymbol{p}_{j}, h_{i j}=\boldsymbol{p}_{i j} \cdot \boldsymbol{n}$, and $\boldsymbol{n}=\left(\boldsymbol{p}_{i}\right.$ $\left.\times \boldsymbol{p}_{j}\right) /\left\|\boldsymbol{p}_{i} \times \boldsymbol{p}_{j}\right\|$, where $\boldsymbol{n}$ is the unit vector normal to the surface. Using the confining-potential approach, ${ }^{2,3}$ we obtained the Schrödinger equation for noninteracting electron systems on curved surfaces as follows:

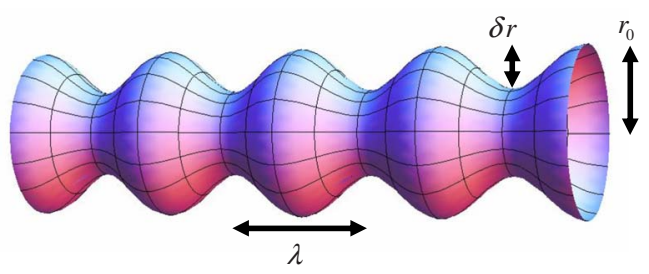

FIG. 1. (Color online) Schematic illustration of a quantum hollow cylinder with periodic radius modulation. 

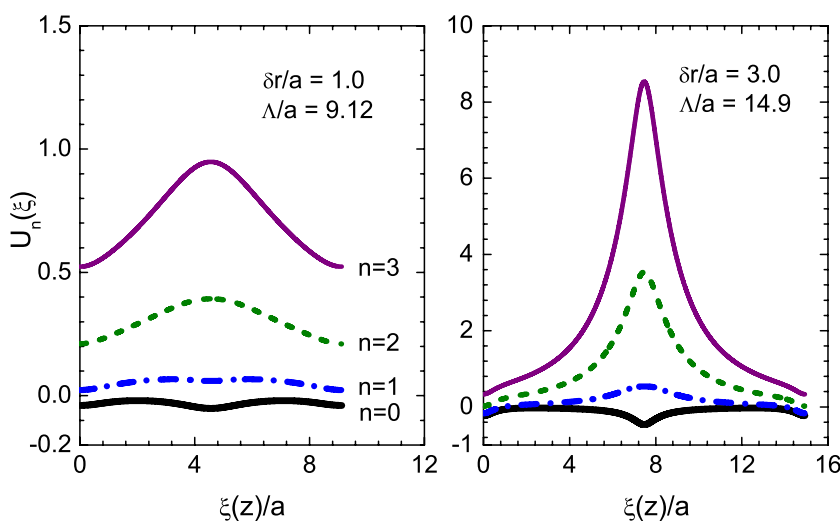

FIG. 2. (Color online) Profiles of the curvature-induced effective potential $U_{n}(\xi)$ for one period $[0, \Lambda]$. Geometric parameters $r_{0}=4.0$ and $\lambda=8.0$ in units of $a$ are fixed. Integers $n$ represent the angular momentum of eigenstates in the circumferential direction of a hollow tube.

$$
-\frac{\hbar^{2}}{2 m^{*}}\left[\frac{1}{\sqrt{g}} \sum_{i, j=1}^{2} \frac{\partial}{\partial u^{i}} \sqrt{g} g^{i j} \frac{\partial}{\partial u^{j}}+\left(\mathcal{H}^{2}-\mathcal{K}\right)\right] \Psi=E \Psi,
$$

where $g=\operatorname{det}\left(g_{i j}\right), g^{i j}=g_{i j}^{-1},{ }^{40}$ and $m^{*}$ is the effective mass of electrons. The quantities $\mathcal{K}=\left(h_{11} h_{22}-h_{12}^{2}\right) / g$ and $\mathcal{H}=\left(g_{11} h_{22}\right.$ $\left.+g_{22} h_{11}-2 g_{12} h_{12}\right) /(2 g)$ are the so-called Gaussian curvature and mean curvature, respectively, both of which are functions of $\left(u^{1}, u^{2}\right)$. The term proportional to $\mathcal{H}^{2}-\mathcal{K}$ in Eq. (1) is the effective scalar potential induced by the surface curvature.

We next focused on a hollow tube with a periodically varying radius represented by $\boldsymbol{p}=[r(z) \cos \theta, r(z) \sin \theta, z]$ (see Fig. 1). The tube radius $r(z)$ is periodically modulated in the axial $z$ direction as $r(z)=r_{0}-\frac{\delta r}{2}+\frac{\delta r}{2} \cos \left(\frac{2 \pi}{\lambda} z\right)$, where the parameters $r_{0}$ and $\delta r$ are introduced to express the maximum and minimum of $r(z)$ as $r_{0}$ and $r_{0}-\delta r$, respectively. Because of the rotational symmetry, the eigenfunctions of the system have the form of $\Psi(z, \theta)=e^{i n \theta} \psi_{n}(z)$. Thus, the problem reduces to the one-dimensional Schrödinger equation

$$
-\frac{\hbar^{2}}{2 m^{*}}\left[\mathcal{D}-\frac{n^{2}}{r^{2}}+\left(\mathcal{H}^{2}-\mathcal{K}\right)\right] \psi_{n}(z)=E \psi_{n}(z),
$$

where $\mathcal{D}=\frac{1}{x f} \frac{d}{d z} \frac{r}{f} \frac{d}{d z}, f(z)=\sqrt{1+r^{\prime 2}}, \mathcal{K}=-r^{\prime \prime} /\left(r f^{2}\right)$, and $\mathcal{H}=\left(f^{2}\right.$ $\left.-r r^{\prime \prime}\right) /\left(2 r f^{3}\right)$ with $r^{\prime} \equiv d r / d z$.

Equation (2) is simplified by using a new variable $\xi=\xi(z)=\int_{0}^{z} f(\eta) d \eta$, which corresponds to the line length along the curve on the surface with a fixed $\theta$. Straightforward calculation yields ${ }^{19}$

$$
\left[-a^{2} \frac{d^{2}}{d \xi^{2}}+U_{n}(\xi)\right] \psi_{n}(\xi)=\varepsilon \psi_{n}(\xi), \quad \varepsilon=\frac{2 m^{*} a^{2} E}{\hbar^{2}}
$$

with $U_{n}(\xi)=\left(n^{2}-\frac{1}{4}\right) a^{2} / r^{2}-r^{\prime \prime 2} a^{2} /\left(4 f^{6}\right)$, where $r, r^{\prime \prime}$, and $f$ are regarded as the functions of $\xi$ using the inverse relation $z$ $=z^{-1}(\xi)$. In order to derive Eq. (3), we introduced the length scale $a$ and then multiplied both sides of Eq. (2) with $2 m^{*} a^{2} / \hbar^{2}$ to make the units of $U_{n}$ and $\varepsilon$ dimensionless. Notice that by the definition of $\xi(z), U_{n}$ is periodic with a period $\Lambda=\xi(\lambda)$ depending on $r_{0}$ and $\delta r$ (as well as $\lambda$ ). Figure 2
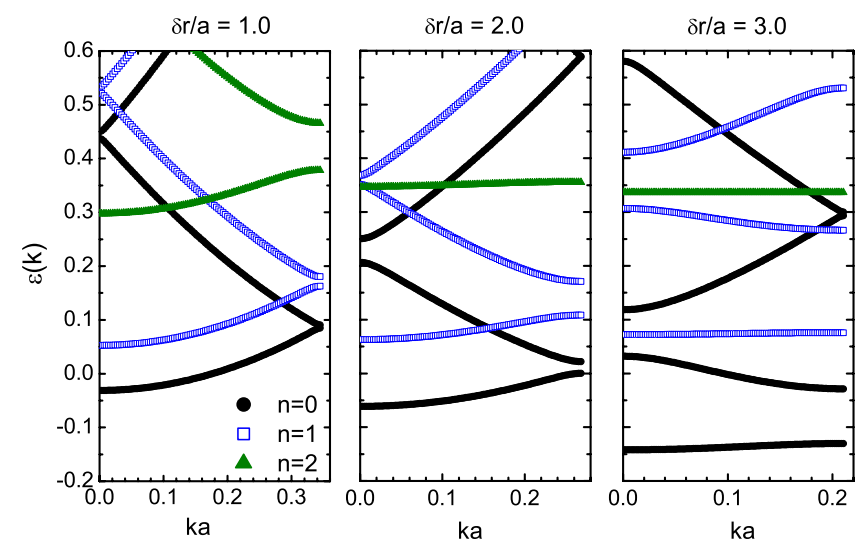

FIG. 3. (Color online) Energy-band structure of a sinusoidal hollow tubule with $r_{0}=4.0$ and $\lambda=8.0$ in units of $a$.

shows the spatial profile of $U_{n}$ within one period; throughout the present work, we fixed $r_{0}=4.0$ and $\lambda=8.0$ in units of $a$ by simulating the geometry of actual peanut-shaped $\mathrm{C}_{60}$ polymers whose geometry is reproduced by imposing $a=1 \AA$. Mapping the discrete atomic structure of one-dimensional $\mathrm{C}_{60}$ polymers to a continuum curved surface is based on the result of first-principle calculations ${ }^{41}$ which indicated that $\pi$ electrons on the polymers are almost free from its atomic configurations. We found in Fig. 2 that $U_{n}$ takes extrema at $\xi=0$ (or $\Lambda$ ) and $\xi=\Lambda / 2$, where $r$ takes the maximum $(r$ $\left.=r_{0}\right)$ and the minimum $\left(r=r_{0}-\delta r\right)$ values, respectively.

To solve Eq. (3), we use the Fourier series expansions $U_{n}(\xi)=\Sigma_{G} U_{G}^{(n)} e^{i G \xi}$ and $\psi_{n}(\xi)=\Sigma_{k} c_{k}^{(n)} e^{i k \xi}$, where $G= \pm 2 \pi j / \Lambda \quad(j=0,1,2, \ldots)$. Substituting the expansions into Eq. (3), we obtain the secular equation $\left(\frac{\hbar^{2}}{2 m^{*}} k^{2}-\varepsilon\right) c_{k}^{(n)}$ $+\sum_{G=-G_{c}}^{G_{c}} U_{G}^{(n)} c_{k-G}^{(n)}=0$ that holds for all possible $k$ 's and $n$ 's. The summation has been truncated by $G_{c}=20 \pi / \Lambda$ because of the rapid decay of $U_{G}$ with $|G|$. We then numerically calculated eigenvalues for $0 \leq k \leq \pi / \Lambda$ and evaluated the lowenergy-band structure for several different $\delta r$, as depicted in Fig. 3. In all the cases, there is some energy gap at the Brillouin-zone boundary $G_{0} \equiv \pi / \Lambda$, where a wider energy gap occurs for a larger $\delta r$, as expected from the large amplitude of $\left|U_{n}(\xi)\right|$ with increasing $\delta r$ (see Fig. 2).

We now consider the Coulombic interactions between spinless electrons. The interactions make the electron-hole pairs share the ground state of the noninteracting electron system, wherein the most strongly affected states are those lying in the vicinity of $E_{F}$. As a consequence, the singleparticle density of states $n(\omega)$ near $E_{F}$ exhibits a power-law singularity of the following form ${ }^{34}$.

$$
n(\omega) \propto\left|\hbar \omega-E_{F}\right|^{\alpha}, \quad \alpha=\frac{K+K^{-1}}{2}-1 .
$$

The explicit form of $K$ is derived using the bosonization procedure $^{34}$ as follows:

$$
K=\lim _{q \rightarrow 0} \sqrt{\frac{2 \pi \hbar v_{F}+g_{4}(q)-g_{2}(q)}{2 \pi \hbar v_{F}+g_{4}(q)+g_{2}(q)}} .
$$

Here, $v_{F}=\hbar^{-1} d E /\left.d k\right|_{k=k_{F}}$ is the Fermi velocity, and $g_{4}(q)$ $=V(q, m)$ and $g_{2}(q)=V(q, m)-V\left(2 k_{F}, m\right)$ are $q$-dependent 
(a)

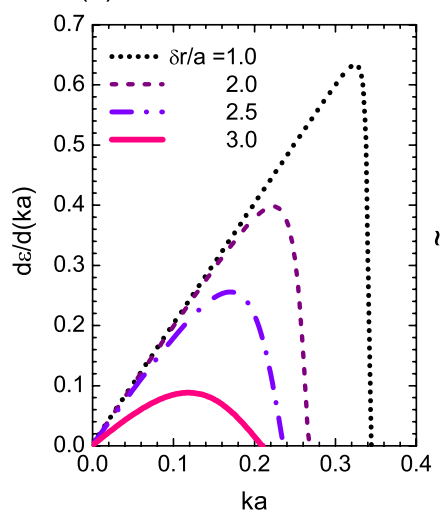

(b)

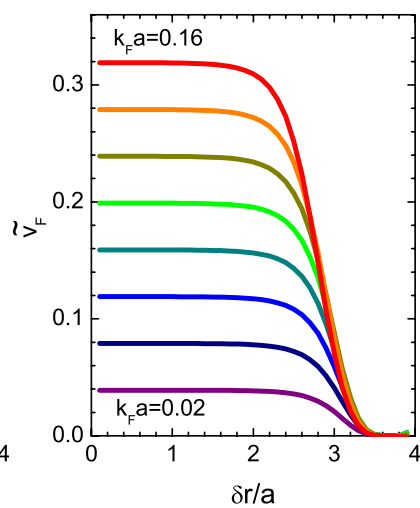

FIG. 4. (Color online) (a) $k$ dependence of the derivative $d \varepsilon / d k$ for the lowest-energy band. (b) $\delta r$ dependence of the dimensionless Fermi velocity $\widetilde{v}_{F}=d \varepsilon /\left.d(k a)\right|_{k=k_{F}}$ for different $k_{F} a$ with increments $\Delta\left(k_{F} a\right)=0.02$.

coupling constants. $V(q, m)$ is the Fourier transform of the screened interaction $V(\boldsymbol{r})=-e^{2} e^{-\kappa|\boldsymbol{r}|} /(4 \pi \varepsilon|\boldsymbol{r}|)$, where $\varepsilon$ is the dielectric constant and $\kappa$ is the screening length. The transformation is performed in terms of the curvilinear coordinates $(\xi, \theta)$, and thus, the resulting $V(q, m)$ becomes a function of both the momentum and angular momentum transfers $q$ and $m$, respectively. To make concise arguments, $E_{F}$ was assumed to lie in the lowest-energy band $(n=0)$. This allows us to eliminate the index $m$ from $V(q, m)$, which leads to $V(q)=-\frac{e^{2}}{4 \pi \varepsilon} \log \left[\left(q^{2}+\kappa^{2}\right) r_{0}^{2}\right]$ for $q r_{0} \ll 1$, in which $r_{0}$ serves as the short-length-scale cutoff.

The aim of the present study is to examine the $\delta r$ dependence of $K$ and $\alpha$, which requires quantification of $k_{F}$ and $v_{F}$. Among the many alternatives, we set $k_{F} a=2.0 \ell \times 10^{-2}(\ell$ $=1, \ldots, 8)$, and evaluate the dimensionless Fermi velocity defined by $\widetilde{v}_{F} \equiv d \varepsilon /\left.d(k a)\right|_{k=k_{F}}$ for each $k_{F}$; the original $v_{F}$ is recovered by the relation $v_{F}=\hbar \tilde{v}_{F} /\left(2 m^{*} a\right)$. We chose the maximum value of $k_{F}$ such that it does not exceed the $\delta r$-dependent zone boundary $G_{0}$ for all $\delta r$. Figure 4(a) shows the plot of $d \epsilon / d k$ as a function of $k a$, from which $\tilde{v}_{F}$ for various $k_{F}$ and $\delta r$ are deduced, as shown in Fig. 4(b). Figure 4(b) shows that $\tilde{v}_{F}$ for all $k_{F}$ remains constant for $\delta r / a$ $<2.0$ but decreases remarkably with increasing $\delta r$ for $\delta r / a$ $>2.5$. The decrease in $\widetilde{v}_{F}$ is caused by the decrease in the slope of dispersion curves $\varepsilon=\varepsilon(k)$ with $\delta r$ (see Fig. 3). The results shown in Fig. 4(b) imply that a change in $\delta r$ leads to a quantitative alteration in $K$ and $\alpha$ for $\delta r / a>2.5$, and furthermore, the degree of alteration increases for larger $k_{F}$, as will be demonstrated later.

Figure 5 shows the $\delta r$ dependence of both $K$ and $\alpha$ for different $k_{F}$ values. According to the bosonization procedure ${ }^{34}$ we set the screening parameter $\kappa$ to be $\kappa a$ $=1.0 \times 10^{-3}$, which is smaller than all $k_{F} a$ values that we have chosen. We also set the interaction-energy scale $e^{2} /(4 \pi \varepsilon a)$ to be 1.1 in units of $\hbar^{2} /\left(2 m^{*} a^{2}\right)$ by simulating that of $\mathrm{C}_{60}$-related materials. ${ }^{42,43}$ The insets in Fig. 5 show the $k_{F}$ dependence of $K$ and $\alpha$ at $\delta r / a=2.0$; each $K$ and $\alpha$ takes the minimum and maximum values, respectively, at the specific $k_{F}$ satisfying the relation $\left(d / d k_{F}\right) \log \left[\left(2 \pi \hbar v_{F}\right.\right.$ $\left.\left.+g_{4}\right) / g_{2}\right]=0$, which is equivalent to $d K / d k_{F}=0$. This result is the same for all $\delta r$ at $\delta r / a<2.0$.
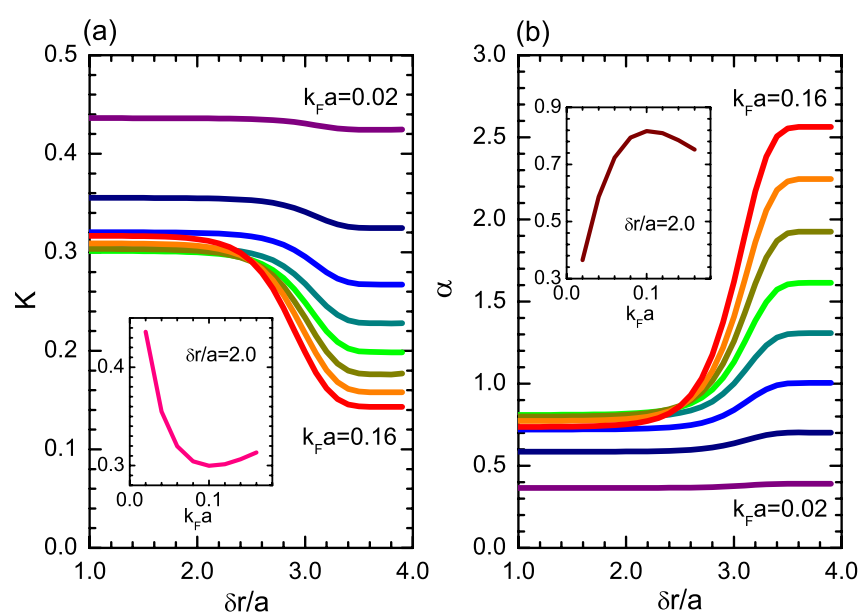

FIG. 5. (Color online) $\delta r$ dependences of $K$ and $\alpha$ defined by Eqs. (4) and (5), respectively. The screening parameter $\kappa$ is set to be $\kappa a=1.0 \times 10^{-3}$. Insets: nonmonotonic behaviors of $K$ and $\alpha$ as a function of $k_{F}$ at $\delta r / a=2.0$.

The salient features of Fig. 5 are the significant decrease in $K$ and increase in $\alpha$ with an increase in $\delta r$ for $\delta r / a$ $>2.5$, as predicted earlier. Such $\delta r$-driven shifts in $K$ and $\alpha$ are attributed to the effects of geometric curvature on the nature of TLL states. In fact, an increase in $\delta r$ amplifies the curvature-induced effective potential $U_{n}(\xi)$, thus yielding a monotonic decrease in $\widetilde{v}_{F}$ at $\delta r / a>2.5$ (see Fig. 4). The decrease in $\widetilde{v}_{F}$ plays a dominant role in the numerator of the expression in Eq. (5) and eventually leads to the systematic shifts in $K$ and $\alpha$. We have confirmed that a change in the value of $\kappa$ does not substantially affect the behaviors of $K$ and $\alpha$ qualitatively, while the absolute values of $K$ and $\alpha$ moderately depends on the choice of $\kappa$. It is noted that Eq. (3) can formally deal with an uncurved one-dimensional system subject to a periodically electrostatic potential. In a similar manner to the present curved system, therefore, the $\alpha$ and $K$ are expected to be shifted even for an uncurved quantum wire by the electric-field modulation.

An important consequence of the results shown in Fig. 5 is that nonzero surface curvature yields diverse alterations in the TLL behaviors of deformed cylinders. This is because various kinds of power-law exponents observed in TLL states are related to the quantity $K$; some such exponents are the exponent of power-law decay in Friedel oscillation ${ }^{44}$ and that of temperature (or voltage)-dependent conductance. ${ }^{45}$ The present theoretical predictions need to be confirmed experimentally, thus opening a field of science that deals with quantum electron systems on curved surfaces.

In conclusion, we reveal that the power-law exponent $\alpha$ of the TLL states in deformed hollow nanocylinders shows a monotonic increase with an increase in the degree of surface curvature. The increase in $\alpha$ is attributed to the curvaturedriven effective potential $U_{n}(\xi)$ that acts on electrons moving along the curved surface. The present results suggest that there are shifts in the power-law exponents of TLL states of real low-dimensional materials such as the peanut-shaped $\mathrm{C}_{60}$ polymers and $\mathrm{MoS}_{2}$.

We acknowledge K. Yakubo, Y. Toda, A. Tokuno, and T. Nishii for stimulating discussions. This study was supported 
by a Grant-in-Aid for Scientific Research from the MEXT, Japan. H.S. is thankful for the financial support from the Executive Office of Research Strategy in Hokkaido Uni- versity. Numerical simulations were carried out using the facilities of the Supercomputer Center, ISSP, University of Tokyo.
*Corresponding author; shima@eng.hokudai.ac.jp

${ }^{1}$ B. De Witt, Phys. Rev. 85, 653 (1952); Rev. Mod. Phys. 29, 377 (1957).

${ }^{2}$ H. Jensen and H. Koppe, Ann. Phys. 63, 586 (1971).

${ }^{3}$ R. C. T. da Costa, Phys. Rev. A 23, 1982 (1981).

${ }^{4}$ L. Kaplan, N. T. Maitra, and E. J. Heller, Phys. Rev. A 56, 2592 (1997).

${ }^{5}$ P. C. Schuster and R. L. Jaffe, Ann. Phys. 307, 132 (2003).

${ }^{6}$ O. G. Schmidt and K. Eberl, Nature (London) 410, 168 (2001).

${ }^{7}$ M. Sano, A. Kamino, J. Okamura, and S. Shinkai, Science 293, 1299 (2001).

${ }^{8}$ S. Tanda, T. Tsuneta, Y. Okajima, K. Inagaki, K. Yamaya, and N. Hatakenaka, Nature (London) 417, 397 (2002).

${ }^{9}$ Y. Oshima, A. Onga, and K. Takayanagi, Phys. Rev. Lett. 91, 205503 (2003).

${ }^{10}$ A. Lorke, S. Bohm, and W. Wegscheider, Superlattices Microstruct. 33, 347 (2003).

${ }^{11}$ D. N. McIlroy, A. Alkhateeb, D. Zhang, D. E. Aston, A. C. Marcy, and M. G. Norton, J. Phys.: Condens. Matter 16, R415 (2004), and references therein.

${ }^{12}$ H. Shima and M. Sato, Nanotechnology 19, 495705 (2008).

${ }^{13}$ T. Fujita, H. Okada, K. Koyama, K. Watanabe, S. Maekawa, and M. W. Chen, Appl. Phys. Lett. 92, 251902 (2008).

${ }^{14}$ I. Arias and M. Arroyo, Phys. Rev. Lett. 100, 085503 (2008).

${ }^{15}$ A. Mostafazadeh, Phys. Rev. A 54, 1165 (1996).

${ }^{16}$ G. Cantele, D. Ninno, and G. Iadonisi, Phys. Rev. B 61, 13730 (2000).

${ }^{17}$ H. Aoki, M. Koshino, D. Takeda, H. Morise, and K. Kuroki, Phys. Rev. B 65, 035102 (2001); M. Koshino and H. Aoki, ibid. 71, 073405 (2005).

${ }^{18}$ M. V. Entin and L. I. Magarill, Phys. Rev. B 66, 205308 (2002).

${ }^{19}$ N. Fujita, J. Phys. Soc. Jpn. 73, 3115 (2004).

${ }^{20}$ J. Gravesen and M. Willatzen, Phys. Rev. A 72, 032108 (2005).

${ }^{21}$ H. Taira and H. Shima, Surf. Sci. 601, 5270 (2007).

${ }^{22}$ V. Atanasov and R. Dandoloff, Phys. Lett. A 372, 6141 (2008).

${ }^{23}$ S. K. Baek, S. D. Yi, and B. J. Kim, Phys. Rev. E 77, 022104 (2008).

${ }^{24}$ D. V. Bulaev, V. A. Geyler, and V. A. Margulis, Phys. Rev. B 69, 195313 (2004).

${ }^{25}$ A. V. Chaplik and R. H. Blick, New J. Phys. 6, 33 (2004).

${ }^{26}$ A. Marchi, S. Reggiani, M. Rudan, and A. Bertoni, Phys. Rev. B
72, 035403 (2005).

${ }^{27}$ G. Cuoghi, G. Ferrari, and A. Bertoni, Phys. Rev. B 79, 073410 (2009).

${ }^{28}$ M. V. Entin and L. I. Magarill, Phys. Rev. B. 64, 085330 (2001); Europhys. Lett. 68, 853 (2004).

${ }^{29}$ E. Zhang, S. Zhang, and Q. Wang, Phys. Rev. B 75, 085308 (2007).

${ }^{30}$ M. Encinosa, Phys. Rev. A 73, 012102 (2006).

${ }^{31}$ O. Olendski and L. Mikhailovska, Phys. Rev. B 72, 235314 (2005); 77, 174405 (2008).

${ }^{32}$ G. Ferrari and G. Cuoghi, Phys. Rev. Lett. 100, 230403 (2008); G. Ferrari, A. Bertoni, G. Goldoni, and E. Molinari, Phys. Rev. B 78, 115326 (2008).

${ }^{33}$ G. Parascandolo, G. Cantele, D. Ninno, and G. Iadonisi, Phys. Rev. B 68, 245318 (2003).

${ }^{34}$ J. Voit, Rep. Prog. Phys. 57, 977 (1994).

${ }^{35}$ J. Onoe, T. Nakayama, M. Aono, and T. Hara, Appl. Phys. Lett. 82, 595 (2003); J. Onoe, T. Ito, S. I. Kimura, K. Ohno, Y. Noguchi, and S. Ueda, Phys. Rev. B 75, 233410 (2007).

${ }^{36}$ Y. Toda, S. Ryuzaki, and J. Onoe, Appl. Phys. Lett. 92, 094102 (2008).

${ }^{37}$ P. Santiago, J. A. Ascencio, D. Mendoza, M. Pérez-Alvarez, A. Espinosa, C. Reza-sangermán, P. Schabes-Retchkiman, G. A. Camacho-bragado, and M. José-Yacamán, Appl. Phys. A 78, 513 (2004)

${ }^{38}$ H. Yoshioka, Physica E 18, 212 (2003).

${ }^{39}$ H. Ishii, H. Kataura, H. Shiozawa, H. Yoshioka, H. Otsubo, Y. Takayama, T. Miyahara, S. Suzuki, Y. Achiba, M. Nakatake, T. Narimura, M. Higashiguchi, K. Shimada, H. Namatame, and M. Taniguchi, Nature (London) 426, 540 (2003).

${ }^{40} \mathrm{H}$. Shima and T. Nakayama, Higher Mathematics for Physics and Engineering (Springer-Verlag, Berlin, 2009).

${ }^{41}$ T. A. Beu, J. Onoe, and A. Hida, Phys. Rev. B 72, 155416 (2005).

${ }^{42}$ A. F. Hebard, R. C. Haddon, R. M. Fleming, and A. R. Kortan, Appl. Phys. Lett. 59, 2109 (1991).

${ }^{43}$ A. Oshiyama, S. Saito, N. Hamada, and Y. Miyamoto, J. Phys. Chem. Solids 53, 1457 (1992).

${ }^{44}$ R. Egger and H. Grabert, Phys. Rev. Lett. 75, 3505 (1995).

${ }^{45}$ C. L. Kane and M. P. A. Fisher, Phys. Rev. B 46, 15233 (1992). 